\title{
Insuficiencia aortica asociada a comunicacion interventricular
}

Clara A Vazquez Antona*

Ricardo Pignatelli**

Ivan Rivera Romero***

\section{Correspondencia}

Clara A Vázquez Antona

vazquezantona@gmail.com

*Departamento de Ecocardiografia Pediátrica. Instituto Nacional de Cardiología Ignacio Chávez, Ciudad de México, México

**Section of Pediatric Cardiology, Department of Pediatrics, Baylor College of Medicine, Texas Children's Hospital, Houston, Texas

***Universidad Federal de Alagoas, Maceió, Brasil

Recibido: 26/02/2021

Aceptado: 28/02/20201

En línea: 30/04/2021

Citar como: Vasquez C., Pignatelli R., Rivera I., Insuficiencia aortica asociada a comunicación interventricular. Rev Ecocar Pract (RETIC). 2021 (Abril); 4 (1): 55-58. doi: 10.37615/retic.v4n1a16.

Cite this as: Vasquez C., Pignatelli R., Rivera I.,Subarterial interventricular communication aortica valve prolapse Two and three dimensional echocardiography Rev Ecocar Pract (RETIC). 2021 (Abril); 4 (1): 55-58. doi: 10.37615/retic.v4n1a16.

\begin{tabular}{l} 
Palabras clave \\
\hline$\triangleright$ Comunicación \\
interventricular subarterial \\
$\triangleright$ Prolapso aórtico \\
$\triangleright$ Ecocardiografía bi \\
y tridimensional \\
\hline \\
Keywords \\
$\triangleright$ Subarterial ventricular septal \\
defect \\
$\triangleright$ Aortic prolapse. \\
$\triangleright$ Bi and three-dimensional \\
echocardiography
\end{tabular}

\begin{tabular}{l} 
RESUMEN \\
En la evolución natural de una comunicación interventricular pueden presentarse alteraciones valvulares \\
aórticas que condicionen insuficiencia aórtica y que modifican el cuadro clínico y pronóstico del paciente, \\
principalmente en las de tipo infundibular subarterial, por lo que es importante definir la anatomía del de- \\
fecto e impacto en la funcionalidad de la válvula, entender que no presentan cierre espontáneo, que requie- \\
re de seguimiento de la evolución de la insuficiencia y determinar el momento del tratamiento quirúrgico, \\
independientemente de la sintomatología. \\
\hline ABSTRACT \\
Aortic valvular abnormalities may occur in the natural history of a ventricular septal defect (VSD), which leads to \\
an aortic insufficiency and modify the clinical picture and prognosis of the patient, mainly in cases of subarterial \\
infundibular type. By this reason it is important to define the anatomy of the defect and its impact on the functio- \\
nality of the valve; understand that they do not present spontaneous closure; requires monitoring and decision of \\
the time of surgical treatment, regardless of the symptoms.
\end{tabular}

No es infrecuente que se presente insuficiencia aórtica (IAo) en la evolución de una comunicación interventricular (CIV), lo que modifica el cuadro clínico y complica el pronóstico y la supervivencia del paciente. Los mecanismos de daño a la válvula aórtica (VAo) son diversos y dependerán del tipo y localización del defecto, así como de sus relaciones anatómicas. Se puede presentar como CIV de tipo perimembranoso, sin embargo, la mayor frecuencia es de tipo infundibular subarterial, en donde se desarrolla entre el 10 al 15\% de los casos, y en población oriental hasta el 30\%1,2. La comunicación interventricular infundibular subarterial (CIVIS), denominada en clasificaciones anteriores como "supracristales o doblemente relacionados", tiene bordes fibromusculares y se localiza debajo de ambas válvulas semilunares, originando continuidad fibrosa entre el anillo pulmonar y aórtico, estando el septum infundibular (SI) parcial o completamente ausente, lo que impide un soporte adecuado de las valvas. Este sustrato anatómico es la causa del desarrollo de prolapso de la valva coronariana (menos frecuente la no coronariana) e IAo.

Se ha propuesto que el mecanismo para el desarrollo de la IAo es la falta de apoyo de la sigmoidea aórtica por la ausencia del SI, principalmente en los defectos grandes. Cuando el defecto es menor existen cambios hemodinámicos a lo largo de todo el ciclo cardíaco: durante la sístole temprana el flujo de alta velocidad condicionado por el cortocircuito a través del defecto tiende a desplazar el anillo sin apoyo hacia abajo y dentro del ventrículo derecho (VD).

Posteriormente, el flujo de salida del ventrículo izquierdo (VI) empuja la valva sin apoyo hacia afuera provocando el prolapso. En diástole, la presión intraaórtica obliga a la VAo a cerrar, pero la cúspide sin soporte es empujada hacia el tracto de salida del VI alejado de la cúspide coronaria opuesta, lo que resulta en insuficiencia. Algunas veces el prolapso de la valva (PAo) ocluye el defecto septal; haciéndolo de menor tamaño y mejorando los síntomas de insuficiencia cardíaca en el paciente.

La progresión de la IAo se incrementa con la edad, generalmente inicia en la edad escolar y puede convertirse en una complicación grave, por lo que algunos grupos prefieren un cierre quirúrgico temprano, el cual debe considerarse cuando se presenta IAo, independientemente de la repercusión hemodinámica de la cardiopatía. También se ha demostrado que este tipo de defecto no presenta cierre espontáneo'. Hacia la tercera década de la vida puede presentarse como aneurisma roto del seno de Valsalva (ASVR). El estudio ecocardiográfico transtorácico (ETT) y transesofágico (ETE) es la técnica no invasiva de elección para la detección y seguimiento de lesiones aórticas asociadas a CIVIS, convirtiéndose en una valiosa ayuda para determinar cuándo realizar el tratamiento quirúrgico ${ }^{2,3}$ 


\section{Evaluación ecocardiográfica}

El estudio irá dirigido a valorar la localización y tamaño del defecto, dirección del cortocircuito, presencia de PAo y/o ASVR, severidad de la IAo, insuficiencia pulmonar y la repercusión hemodinámica sobre cavidades izquierdas, así como presión pulmonar.

- La localización y tamaño CIVIS, así como en PAo se valoran en eje largo y corto paraesternal, ya que es en estas aproximaciones donde se observa el SI (Figura 1, Video 1). En eje largo es posible ver la valva coronariana derecha deformada y prolapsando hacia el VD y definir si cierra parcialmente el defecto e incluso en su caso, observar ASVR (Figura 2, Video 2). En eje corto paraesternal el defecto se sitúa entre las 12 y 2 (imaginando la carátula del reloj) y se observa además la continuidad entre las sigmodeas aórticas y pulmonares. La CIV perimembranosa se localiza entre las 9 y 12 y en relación con la válvula tricúspide. También es posible determinar la deformación de la valva coronariana derecha en diástole cuando prolapsa. Con Doppler color se localiza el defecto y la dirección del cortocircuito.

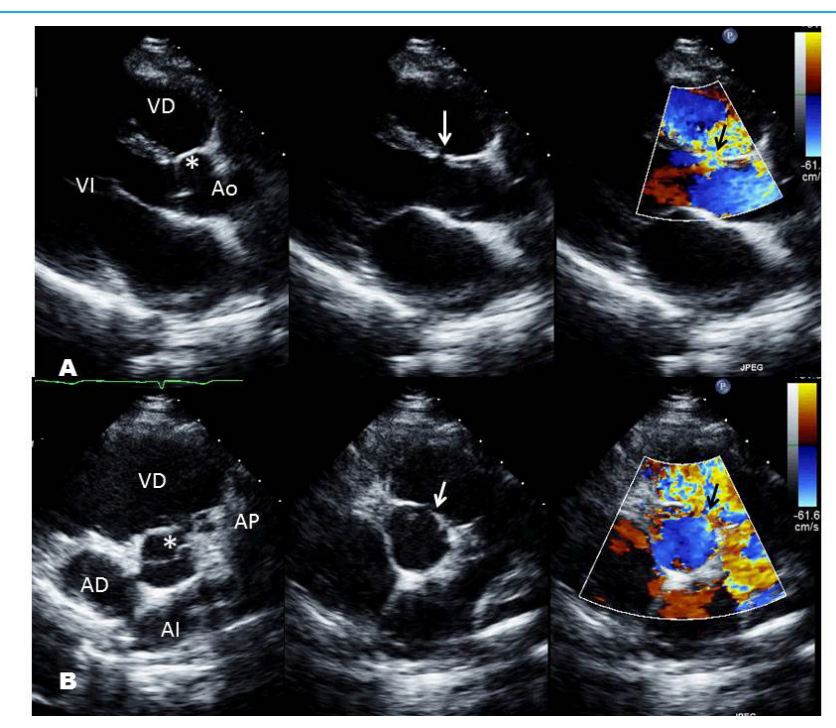

Figura 1. Imágenes de ecocardiograma transtorácico bidimensional (ETT 2D) de una Comunicación interventricular infundibular subarterial (CIVIS) pequeña con prolapso aórtico (PAo). En el panel superior (A) se observa en eje largo paraesternal (ELPE), en diástole, el PAo (asterisco), la CIVIS (flechas) y, con Doppler color, el cortocircuito de izquierda a derecha. En el panel inferior (B) se muestra el eje corto paraesternal (ECPE), obsérvese la deformación de la valva coronariana derecha por el prolapso, la localización del defecto (entre las 12 y 2) y la continuidad de las sigmoideas aórticas y pulmonares. AD: aurícula derecha, Al: aurícula izquierda, VD: ventrículo derecho, Vl: ventrículo izquierdo, Ao: aorta, AP: arteria pulmonar

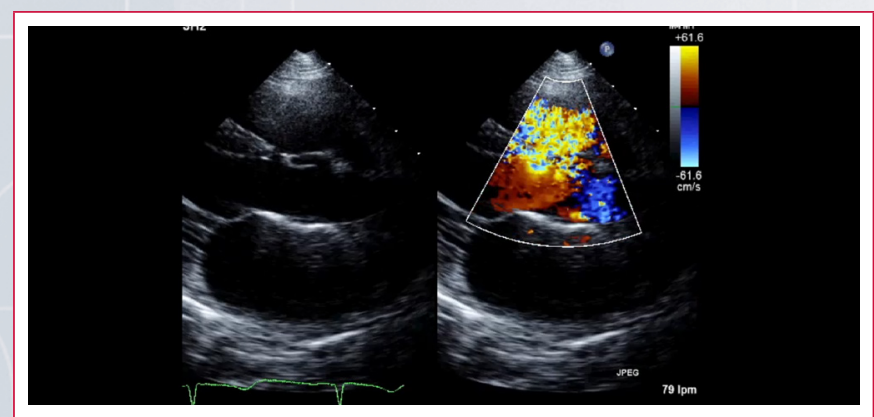

Vídeo 1. Ecocardiograma transtorácico bidimensional (ETT 2D) de una Comunicación interventricular infundibular subarterial (CIVIS) pequeña con prolapso aórtico (PAo). Se observa en eje largo paraesternal (ELPE), en diástole, el PAo, la CIVIS y, con Doppler color, el cortocircuito de izquierda a derecha

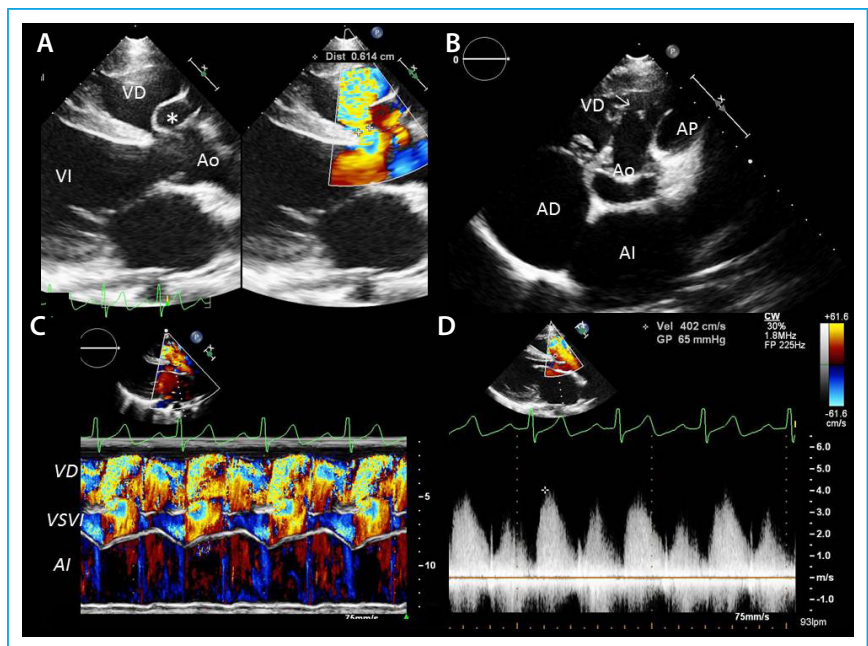

Figura 2. . Imágenes ETT 2D de CIVIS con PAo y aneurisma del seno de Valsalva roto (ASVR) hacia el ventrículo derecho (VD). (A): Imagen en ELPE; que muestra el PAo (asterisco) que cierra parcialmente el defecto. (B): Obsérvese además la continuidad de las sigmoidea aórticas y pulmonares que caracterizan a estos defectos y la flecha señala ASVR. (C): Modo M color en ELPE. obsérvese el flujo dirigiéndose hacia la CIV (color rojo) en sístole dentro de la vía de salida de ventrículo izquierdo (VSVI) y dentro del VD convergen el flujo que genera el cortocircuito de la CIVIS y el flujo del ASVR (mosaico de colores), el que se mantiene en la diástole. (D): Doppler continuo que cuantifica gradiente de la CIVIS. AD: aurícula derecha, Al: aurícula izquierda, VD: ventrículo derecho, VI: ventrículo izquierdo, Ao: aorta, AP: arteria pulmonar

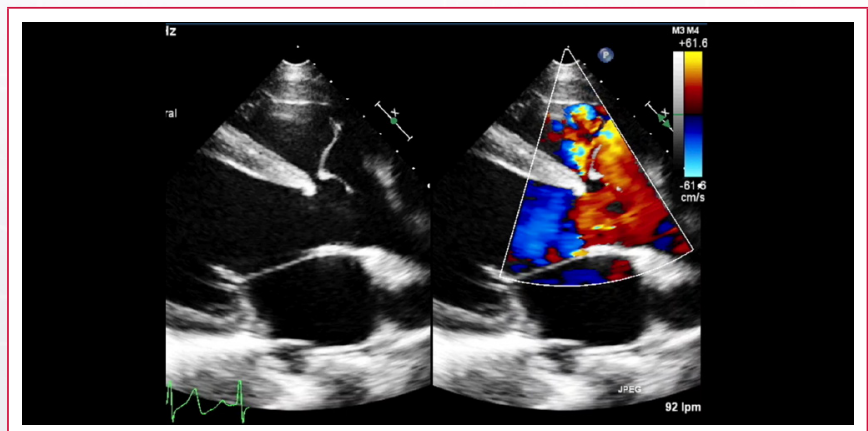

Vídeo 2. ETT 2D de una CIVIS pequeña con PAo. Se observa en ELPE la deformación de la valva coronariana derecha por el prolapso, la localización del defecto y la continuidad de las sigmoideas aórticas y pulmonares

El gradiente de la CIVIS se mide con Doppler continuo, con lo que se estima la presión pulmonar restando este de la presión sistólica sistémica en ausencia de obstrucción aórtica. Con Modo M color es posible comprender el comportamiento de las lesiones durante el ciclo cardíaco (Figuras 1 y 2). La presencia y repercusión de IAo se valora con Doppler pulsado, continuo y color, en aproximación apical de 4 y 5 cámaras (4C, 5C). Entre los parámetros que indican severidad están: falta de coaptación de las valvas, espectro con Doppler continuo denso y completo, el ancho del flujo regurgitante con Doppler color en la vía de salida de VI > 65\%, flujo diastólico reverso en aorta descendente a nivel del istmo con velocidad $>20 \mathrm{~cm} / \mathrm{seg}$ y tiempo de hemipresión (THP) < 200 ms; en pacientes adolescentes y adultos, además, vena contracta $>6 \mathrm{~mm}$, volumen regurgitante $>60 \mathrm{ml}$, fracción regurgitante $>50 \%$ y dilatación de VI (Figura 3, Video 3 ).

Se deberá evaluar repercusión hemodinámica sobre VI: tamaño y función y la presión pulmonar.

Las vistas que se logran con eco tridimensional (3D) y 3D color valoran la anatomía y repercusión de las lesiones, en particular las vistas de superficie septal en donde se puede definir con precisión el tamaño real de la CIVIS o la reconstrucción 3D de la VAo (Figura 4, Video 4). 


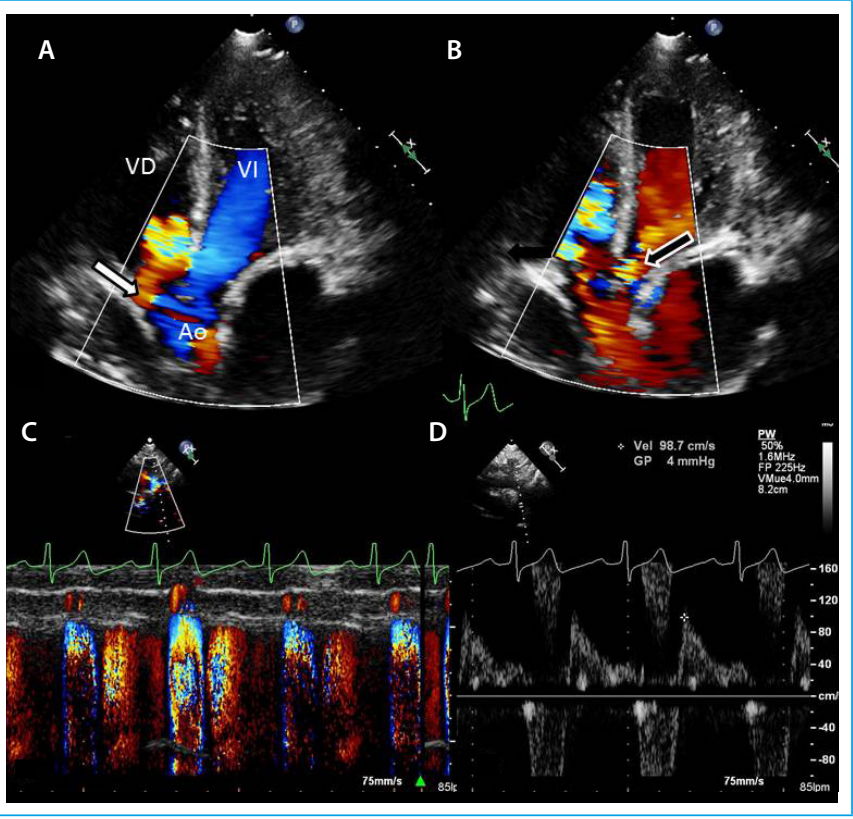

Figura 3. Imágenes ETT 2D que muestran en aproximación apical de 5 cámaras (5C) en sístole (A) el flujo del ASVR hacia VD (flecha blanca) que continua hasta la diástole (B) en donde además aparece en ventrículo izquierdo (VI) el flujo de la insuficiencia aórtica (IAo) que es severa (flecha negra) y que se corrobora con Modo M color en aorta descendente, en donde se observa el flujo diastólico reverso durante toda la diástole (C) y con Doppler pulsado una velocidad $>20 \mathrm{~cm} / \mathrm{seg}$ (D). VD: entrículo derecho, Vl: ventrículo izquierdo, Ao: aorta

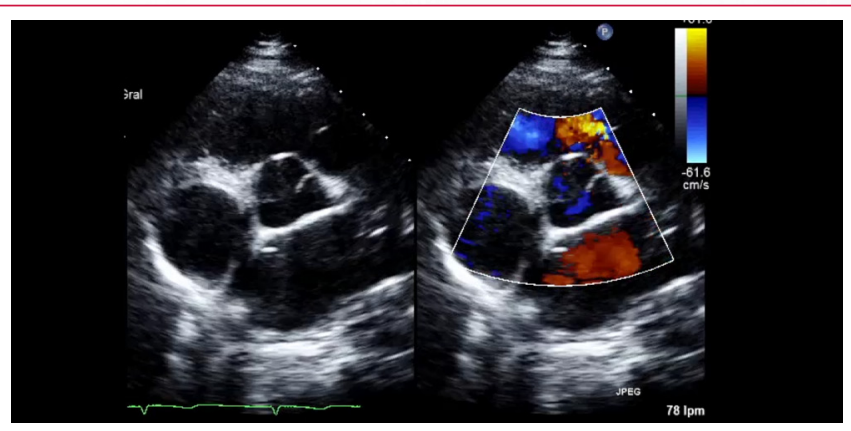

Vídeo 3. ETT 2D de una CIVIS pequeña con PAo. En el panel inferior (B) se muestra el eje corto paraesternal (ECPE), obsérvese la deformación de la valva coronariana derecha por el prolapso, la localización del defecto (entre las 12 y 2) y la continuidad de las sigmoideas aórticas y pulmonares

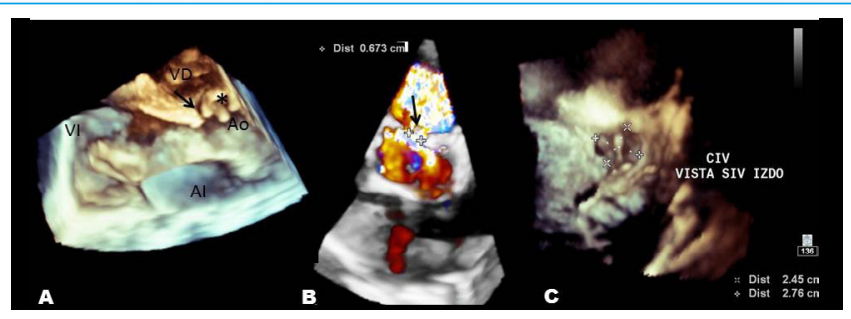

Figura 4. Imágenes de ecocardiograma transtorácico tridimensional (ETT 3D) y 3D color que muestran en reconstrucciones desde el ELPE, con inclinación, el PAo (asterisco) que ocluye parcialmente la CIVIS (flecha), permitiendo un defecto "funcional" de $6.7 \mathrm{~mm}$ (A y B). Al reconstruir la vista de superficie septal desde el VI y "quitar" la valva coronariana derecha (C) se observa que el tamaño real del defecto es de $24 \times 27 \mathrm{~mm}$ en un paciente 16 años con anillo aórtico de $28 \mathrm{~mm}$. Al: aurícula izquierda, VD: ventrículo derecho, VI: ventrículo izquierdo, Ao: aorta, CIA: comunicación interventricular, SIV IZDO: septum interventricular izquierdo

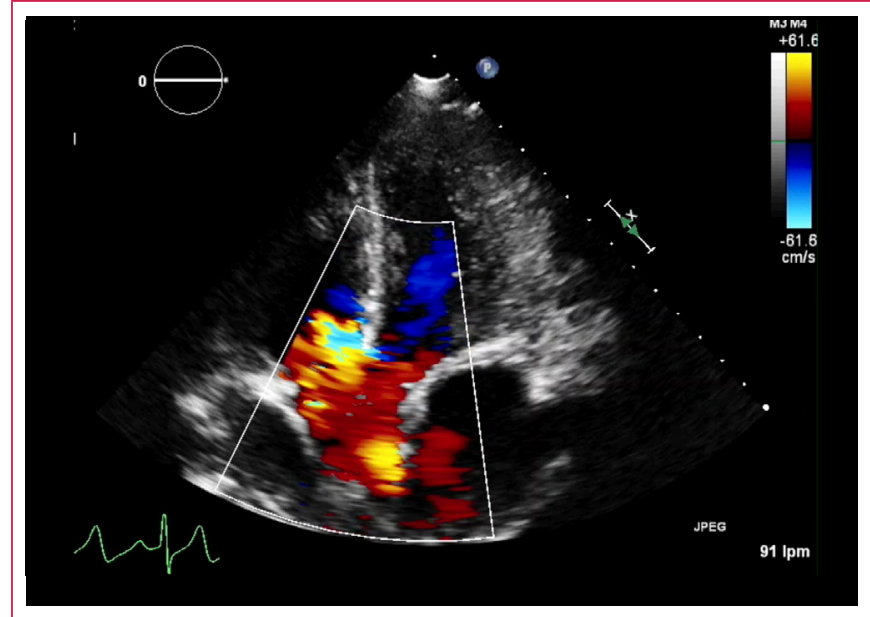

Vídeo 4. 2D que muestran en aproximación apical de 5 cámaras (5C) en sístole el flujo del ASVR hacia VD que continua hasta la diástole, en donde además aparece en ventrículo izquierdo (VI) el flujo de la insuficiencia aórtica (IAo) que es severa

La ETE intraoperatoria confirma el diagnóstico y valora los resultados del cierre del defecto y de la reparación de la VAo con la finalidad de detectar lesiones residuales que ameriten reintervención. La CIVIS se observa en esófago medio en vista de entrada y salida de VD a 60-70', por debajo de la valva coronariana derecha y en relación a las sigmoideas pulmonares, también se puede observar la VAo y el PAo, además de en $45^{\circ}$ y $120^{\circ}-140^{\circ}$ longitudinal de 3 cámaras (Figura 5, Video 5). La IAo se cuantifica con Doppler en transgástrico 0 a $20^{\circ}$.

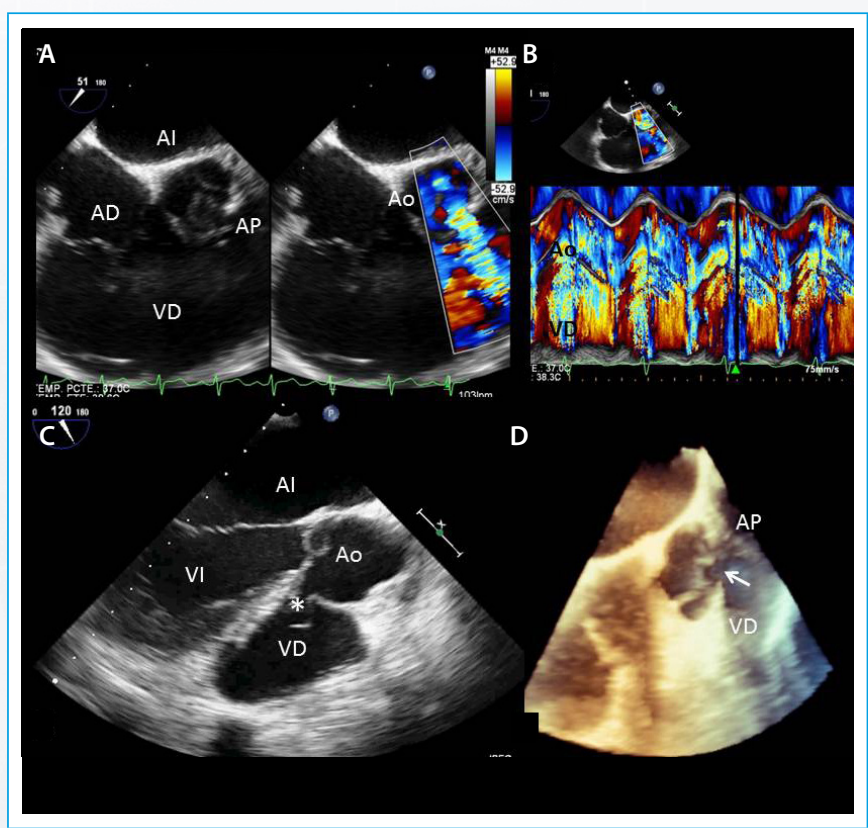

Figura 5. Imágenes de ecocardiograma transesofágico (ETE) que muestran una CIVIS pequeña y PAo. A. ETE medio esofágico a $50^{\circ}$ y C. $120^{\circ}$, obsérvese la deformación de la valva coronariana derecha por el prolapso (asterisco) y el cortocircuito a través del defecto septal situado debajo de esta valva y en continuidad con las sigmoideas pulmonares. B. Modo M color sobre válvula aórtica y VD, en donde se demuestra el flujo de alta velocidad protosistólico producido por el cortocircuito arteriovenoso de la CIVIS. D. Imágenes de reconstrucción 3D en donde se observa el defecto septal (flecha). AD: aurícula derecha, Al: aurícula izquierda, VD: ventrículo derecho, VI: ventrículo izquierdo, Ao: aorta, AP. arteria pulmonar 


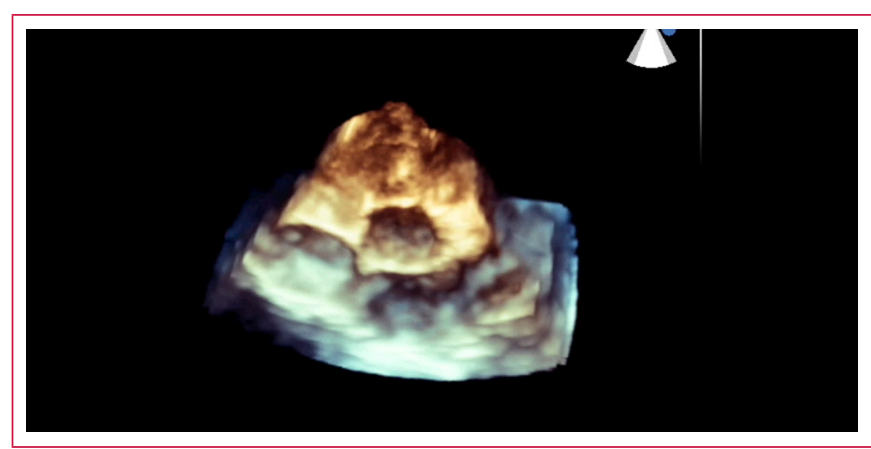

Vídeo 5. Ecocardiograma transesofágico tridimensional (ETE 3D) que muestra una CIVIS pequeña y PAo

\section{Ideas para recordar}

- La comunicación interventricular infundibular subarterial (CIVIS) no evoluciona al cierre espontáneo y como parte de su evolución natural desarroIlan PAo e IAo progresiva.

- En la CIVIS el cortocircuito puede ser variable independiente del tamaño del defecto ya que el PAo puede reducirlo.

- Las CIVIS pequeñas evolucionan a grados más graves de IAo en menor tiempo y en el paciente adulto joven puede presentarse como ASVR.
- El estudio ecocardiográfico ha demostrado ser la técnica de elección para la detección y seguimiento de lesiones aórticas asociadas a CIVIS.

- El ecocardiograma deberá detallar el tipo y localización del defecto y diferenciarla de la CIV perimembranosa (erróneamente llamadas "subaórticas"), así como determinar la presencia de prolapso e IAo y establecer un plan de seguimiento con la finalidad de determinar el momento quirúrgico, independiente de la sintomatología del paciente.

\section{Bibliografía}

1. Hernández Morales G, Vázquez-Antona CA, Muñoz Castellanos L, Vallejo M. Romero A et al: Alteraciones aórticas asociadas a comunicación interventricular infundibular subarterial. Seguimiento ecocardiográfico. Rev Esp Cardiol 2002;55(9):936-42.

2. Jung $\mathrm{H}$, Cho J, Lee Y. Progression of Aortic Regurgitation After Subarterial Ventricular Septal Defect Repair: Optimal Timing of the Operation. Pediatr Cardiol. 2019 Dec;40(8):1696-1702. doi: 10.1007/s00246-019-02206-Z.

3. Yacoub M, Khan H, Stavri G, Shinebourne E, Radley-Smith R. Anatomic correction of the syndrome of prolapsing right coronary aortic cusp, dilatation of the sinus of Valsalva, and ventricular septal defect. J Thorac Cardiovasc Surg 1997;113:253-61. 